\title{
Supply Chain Complex Network Optimization Model Based on Intelligent Algorithm
}

\author{
Chunyan Qiu, ${ }^{1,2, a}$, Song Jiang ${ }^{1}$ and Yang Liu ${ }^{1, b, *}$ \\ ${ }^{1 .}$ School of Management Science and Information Engineering, Jilin University of Finance and Economics, \\ Changchun 130117, China \\ ${ }^{2}$ Key Laboratory of Logistics Industry Economy and Intelligent Logistics, Jilin University of Finance and \\ Economics, Changchun 130117 China \\ aemail:81692002@qq.com, bemail: 7923759@qq.com \\ *Corresponding author
}

Keywords: Artificial Bee Colony Algorithm, Supply Chain, Complex Network

\begin{abstract}
Enterprise supply chain optimization is to find the best decision-making solution that meets the enterprise benefits from various combinations of different nodes in the chain. Intelligent optimization algorithm can solve this problem well. Supply chain optimization based onintelligent optimization algorithm can provide an important reference for enterprise decision-making. Based on the complex network, this paper summarizes the complex network characteristics of supply chain, and focuses on the optimization of supply chain by the artificial bee colony algorithm.
\end{abstract}

\section{Introduction}

With the development of Internet technology, the number of suppliers of raw materials and components has soared, and the supply chain network (SCN) has become more and more complicated. Manufacturers aim to deliver high quality products and services to customers at the lowest cost and least response time. Thereforeoptimizing their supply chain network becomes a huge challenge.

Existing literatures have proposed supply chain designs using various algorithms to solve the dual objective optimization problem. For example, Shaw et al.[1] proposed a method for selecting suitable suppliers in a supply chain network using fuzzy analytic hierarchy process and fuzzy multi-objective linear programming, without considering the structural importance of complex networks. Yeh and Chuang[2] introduced green standards as a framework for supplier selection criteria, regardless of heuristic algorithms.Moncayo-martinez and Zhang[3] proposed an ant colony algorithm-based approach to ensure product delivery without delay through group optimization that aim at minimizing total supply chain costs and lead times. They pointed out that the ant colony algorithm can achieve dual-objective optimization. But the search speed for SCN complexity needs to be improved. Yuce et al. [4] used the artificial bee colony algorithm to optimize the dual-target supply chain model. For a given SCN problem, it can achieve both a minimum total cost and a minimized total response time, but it cannot be determined that the final solution obtained is a global optimal solution.

In order to solve the above problems, this paper integrates the idea of complex network into the artificial bee colony algorithm, and adds the idea of simulated annealing to increase the global search optimization solution set in the supply chain network, which makes up for the shortcomings of the artificial bee colony algorithm that is easy to fall into local optimum. In order to improve the speed of searching global POS, the idea of gradient descent is adopted to guide the random solution to the global optimal solution. The reference of Bayesian probability updates the selection probability of each node in the SCN. 


\section{Related theory}

\subsection{Artificial bee colony algorithm}

Artificial bee colony algorithm is an optimization method which imitates bee behavior.It does not need to understand the special information of the problem. It just needs to compare the pros and cons of the problem. Through the local optimization behavior of each artificial bee individual, the global optimal value is finally emerged in the group, which has a faster convergence speed.

\subsection{Gradient descent algorithm}

Gradient descent algorithm is an optimization algorithm for solving extremum along the direction of gradient descent, It uses a iterative method to find a local optimal solution. The principle is to use the negative gradient direction as the search direction and recursively approximate the extremum according to the selected step size. The convergence speed of the algorithm is faster in the initial stage.

\subsection{Simulated annealing algorithm}

The simulated annealing algorithm is a generalized probability algorithm for finding the optimal solution of a proposition in a large search space. At a given initial temperature, an approximate optimal solution can be obtained in polynomial time by slowly dropping the temperature parameters. It has asymptotic convergence and parallelism.

\subsection{Complex network}

A complex network is a network with some or all properties inself-organizing, self-similarity, attractors, small worlds or scale-free. For the first time, it uses the concept of "degree" to express the connection between substances. It points out that nodes of the same cluster in the network are densely connected and nodes of different clusters are sparse.

\section{Construction of supply chain complex network optimization model based on intelligent algorithm}

By introducing the search method of gradient descent algorithm which advance along a negative gradient direction into the artificial bee colony algorithm, the solution and selection method of proximity solutions of artificial bee colonies can be changed, and the optimization speed of the artificial bee colony algorithm is accelerated to some extent. The parameters such as temperature $(\mathrm{T})$ and annealing speed of the simulated annealing algorithm are difficult to control. The combination of this algorithm and artificial bee colony algorithm can make up for the shortage of artificial bee colony algorithm and gradient descent algorithm which rely too much on initial solution set and easily fall into local optimum. Moreover, good parallel capability can realize parallel operation to speed up the optimization when solving the optimization problem of supply chain management and decision-making. A large number of studies have shown that the artificial bee colony algorithm has a good performance in solving many optimal combination problems which have different data sets and different objective functions [5,6], but the artificial bee colony algorithm is still easy to fall into the local optimal solution.

Based on this situation, the model is mainly based on the artificial bee colony algorithm, using the relevant technology of complex networks to strengthen the interconnection between supply chain structures and simplifying the supply chain decision solving process. Adding the idea of gradient descent and simulated annealing, the rate of convergence is accelerated as much as possible while greatly expanding the search result set of the artificial bee colony algorithm. The model adjusts the optimization times of the algorithm by the temperature $(\mathrm{T})$ in the simulated annealing and uses the Metropolis criterion in simulated annealing to dynamically determine whether to accept a new solution.At the same time, we introduce Bayesian probability, which can maximize the utility of each randomly obtained solution, improve the illumination of the original 
bee colony algorithm, and realize the potential directivity of each solution that is obtained for each cycle to the global optimal solution, so as to speed up the solving speed of the algorithm. Therefore, the algorithm proposed in this paper can achieve faster convergence speed while better dealing with supply chain decision problems of complex structures (especially when the amount of data is particularly large). Therefore, under the condition of improving the service quality of enterprises, the algorithm greatly reduces the cost of the enterprise and achieves the goal of sustainable development of green. The supply chain complex network optimization model based on intelligent algorithm is shown in figure 1.

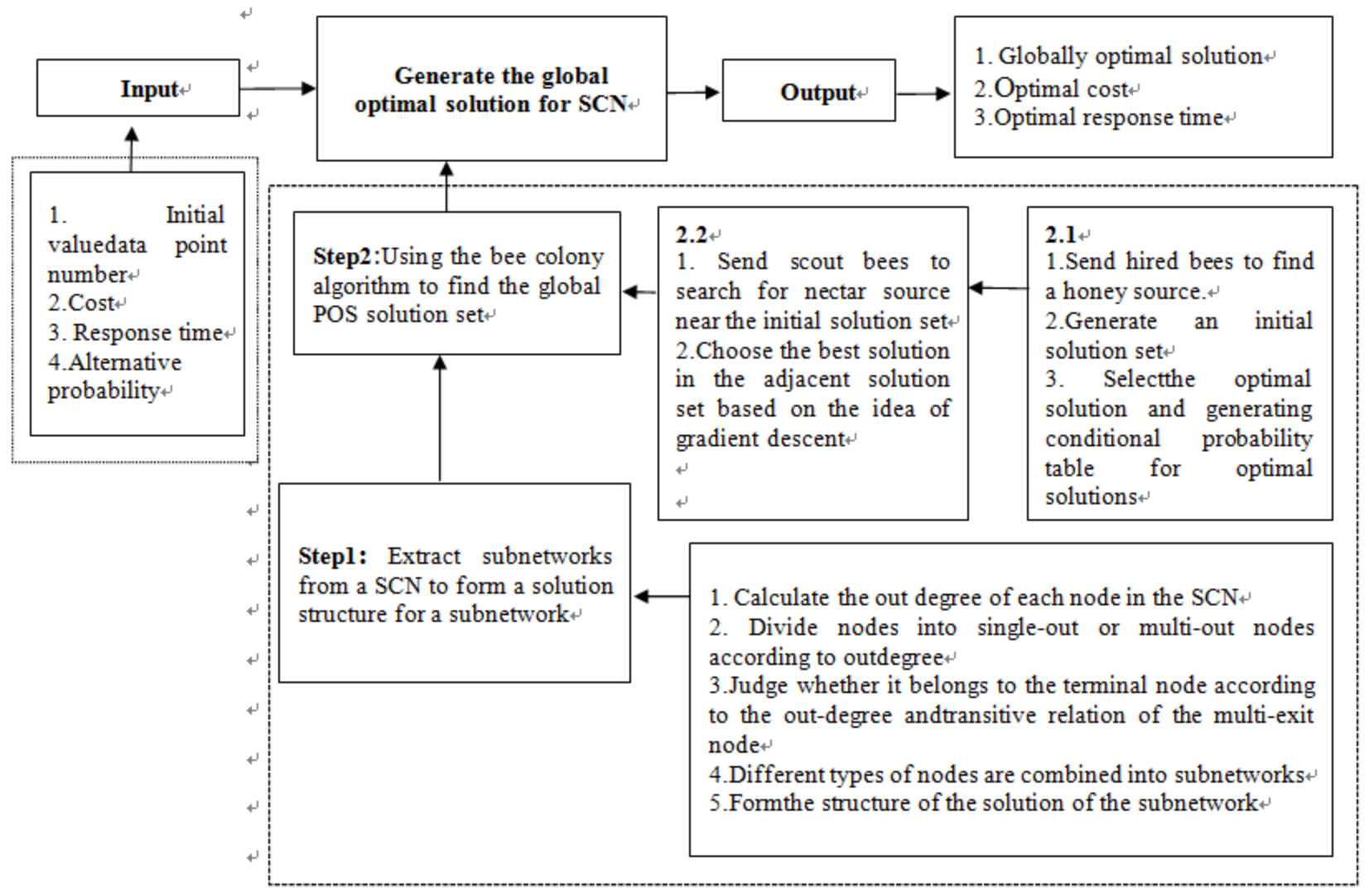

Figure 1: supply chain complex network optimization model based on intelligent algorithm

\section{Conclusions}

In this paper, the advantages of gradient descent and simulated annealing algorithm are added into artificial bee colony algorithm, which can avoid the disadvantages of artificial bee colony algorithm effectively. The introduction of complex networks and Bayesian probability ideas has narrowed the scope of global optimal solutions and accelerated the optimization of the entire algorithm. Based on this, when enterprise decision makers show a clear tendency to certain indicators, using algorithms can help them quickly and efficiently obtain supply chain decision-making solutions. The next step is to conduct simulation experiments on the supply chain complex network optimization model based on intelligent algorithm to further verify the effectiveness of the algorithm.

\section{Acknowledgements}

Funding: This study was funded by Jilin Province Education Science"the13th Five-Year Plan " annual issue in 2017 (Grant Number GH170336).

\section{References}

[1] Shaw K, Shankar R, Yadav S S, et al. Supplier Selection Using Fuzzy AHP and Fuzzy 
Multi-objective Linear Programming for Developing Low Carbon Supply Chain [J]. Expert Systems with Applications, 2012, 39(9):8182-8192.

[2] Yeh W C and Chuang M C. Using Multi-objective Genetic Algorithm for Partner Selection in Green Supply Chain Problems[J]. Expert Systems with Applications, 2011, 38(4):4244-4253.

[3] Moncayo-Martínez L A and Zhang D Z. Optimising Safety Stock Placement and Lead Time in an Assembly Supply Chain Using Bi-objective MAX-MIN Ant System [J]. International Journal of Production Economics, 2013, 145(1):18-28.

[4] Yuce B, Mastrocinque E, Lambiase A. A Multi-Objective Optimization for Supply Chain Network Using the Bees Algorithm[J]. International Journal of Engineering Business Management, 2013, 5:38(1):1-11.

[5] Daniel J S R and Rajendran C. A Simulation-based Genetic Algorithm for Inventory Optimization in a Serial Supply Chain [J]. International Transactions in Operational Research, 2010, 12(1):101-127.

[6] Gong M, Liang Y, Shi J, et al. Fuzzy C-means Clustering with Local Information and Kernel Metric for Image Segmentation [J]. Image Processing IEEE Transactions on, 2013, 22(2):573-84. 